\title{
Non-toxic sulfur inhibits LPS-induced inflammation by regulating TLR-4 and JAK2/STAT3 through IL-6 signaling
}

\author{
DONG YOUNG KANG ${ }^{1 *}$, NIPIN SP ${ }^{1 *}$, EUN SEONG JO ${ }^{1}$, ALEXIS RUGAMBA ${ }^{1}$, HYOUNG DO KIM $^{2}$, \\ IL HO KIM ${ }^{2}$, JONG-CHAN PARK ${ }^{3}$, SE WON BAE ${ }^{4}$, KYOUNG-JIN JANG $^{1}$ and YOUNG MOK YANG ${ }^{1}$ \\ ${ }^{1}$ Department of Pathology, School of Medicine, Institute of Biomedical Science and Technology, Konkuk University, \\ Chungju, North Chungcheong 27478; ${ }^{2}$ Nara Bio Co., Ltd., Gunsan, Jeollabuk-do 54006; ${ }^{3}$ Plant Genome Research Center, \\ Korea Research Institute of Bioscience and Biotechnology, Yuseong, Daejeon 34141; \\ ${ }^{4}$ Department of Chemistry and Cosmetics, Jeju National University, Jeju-si, Jeju-do 63243, Republic of Korea
}

Received April 22, 2020; Accepted March 29, 2021

DOI: $10.3892 / \mathrm{mmr} .2021 .12124$

\begin{abstract}
Janus kinase 2(JAK2) and STAT3 signaling is considered a major pathway in lipopolysaccharide (LPS)-induced inflammation. Toll-like receptor 4 (TLR-4) is an inflammatory response receptor that activates JAK2 during inflammation. STAT3 is a transcription factor for the pro-inflammatory cytokine IL-6 in inflammation. Sulfur is an essential element in the amino acids and is required for growth and development. Non-toxic sulfur (NTS) can be used in livestock feeds as it lacks toxicity. The present study aimed to inhibit LPS-induced inflammation in $\mathrm{C} 2 \mathrm{C} 12$ myoblasts using NTS by regulating TLR-4 and JAK2/STAT3 signaling via the modulation of IL-6. The 3-(4,5-dimethylthiazol-2-yl)-2,5-diphenyltetrazolium bromide assay was conducted to analyze cell viability and reverse transcription polymerase chain reaction and western blotting performed to measure mRNA and protein expression levels. Chromatin immunoprecipitation and enzyme-linked immunosorbent assays were used to determine the binding activity of proteins. The results indicated that NTS demonstrated a protective effect against LPS-induced cell death and inhibited LPS-induced expression of TLR-4, JAK2, STAT3 and IL-6. In addition, NTS inhibited the expression of nuclear phosphorylated-STAT3 and its binding to the IL-6 promoter. Therefore, NTS may be a potential candidate drug for the treatment of inflammation.
\end{abstract}

Correspondence to: Professor Young Mok Yang or Professor Kyoung-Jin Jang, Department of Pathology, School of Medicine, Institute of Biomedical Science and Technology, Konkuk University, Building No. U2, 268 Chungwon-daero, Chungju, North Chungcheong 27478, Republic of Korea

E-mail: ymyang@kku.ac.kr

E-mail: jangkj@konkuk.ac.kr

*Contributed equally

Key words: non-toxic sulfur, Toll-like receptor 4, Janus kinase 2, STAT3, IL-6

\section{Introduction}

Inflammation is considered a localized immune response against harmful or irritating stimuli that activate the immune system. Generally, the immune system recognizes stimuli that cause inflammation and induces an immune response depending on the type of stimuli (1). In a cell culture system, lipopolysaccharide (LPS), a component in the outer membrane of gram-negative bacteria, is used to induce inflammation (2). It induces inflammatory responses by regulating Toll-like receptor (TLR) signaling (3). Among the TLRs, TLR-2 and TLR-4 are considered to serve key roles in inflammation, which subsequently activates NF- $\mathrm{KB}$ signaling $(4,5)$. In response to LPS-induced inflammation, NF- $\kappa B$ activates pro-inflammatory cytokines, such as TNF- $\alpha$, IL-1 $\beta$, or IL-6 (6-8).

The Janus kinase (JAK) family of protein tyrosine kinases takes part in various cellular responses against stimuli, including cancer (9). JAK2 is a ligand that binds to the cell membrane receptor to trigger downstream signaling events from the receptor to the target molecule. Once a stimulus activates the receptor, tyrosine phosphorylation of JAK2 occurs, which signals to other downstream targets. JAK2 is involved in LPS-induced inflammation (10). In particular, LPS-induced inflammation activates TLR-4, triggering the phosphorylation of JAK2, which is required for the activation of various signaling pathways, including Src-kinase, MAPK, PI3K-AKT and STAT (11).

Of the JAK2-mediated signaling pathways, STAT3 serves an important role in inflammation (12). STAT3 is also considered an oncogene and it can transduce signals from stimuli at the cell surface to the nucleus (13). When JAK2 is phosphorylated, the Src-homology 2 domain of STAT3 binds to JAK2, leading to the phosphorylation of STAT3; this in turn translocates to the nucleus and binds to gene promoters to initiate transcription (14). When inflammation occurs, STAT3 activation leads to its binding to the promoter of IL-6, which is a pro-inflammatory cytokine and key mediator in inflammation (15). A recent study has shown that the JAK2/STAT3 signaling pathway is required for cell death and inflammation in pancreatic cells (16). In addition, elevated expression levels of JAK2/STAT3/IL-6 are involved in inflammation during 
tumorigenesis (17) and increased production of IL- 6 has also been identified in cancer (18). Therefore, focusing on the JAK2/STAT3/IL-6 pathway for inflammation may provide a target to develop an anti-inflammatory drug.

A number of sulfur compounds are known to exhibit anti-inflammatory activities (19). Methylsulfonylmethane (MSM) acts as an anti-inflammatory drug against LPS-induced inflammatory responses (20). Sulfur is an essential element that is indirectly consumed by our body in the form of foods, such as onion, garlic and duck meat (21). For the consumption of naturally existing mineral sulfur, different substances are combined with mineral sulfur to eliminate its toxic substances. A number of countries use a non-toxic form of dietary sulfur or natural sulfur for the treatment of different diseases, including inflammatory diseases (22). Non-toxic sulfur (NTS) has been used in livestock feed to improve meat quality and immunity. It has been reported that repeated oral administration of NTS in rats did not induce cell death $(23,24)$. In addition, it has been demonstrated that NTS can enhance growth hormone signaling by regulating the JAK2/STAT5b/IGF-1 axis (25). The present study hypothesized that NTS exhibits anti-inflammatory activity against LPS-induced inflammation by regulating JAK2/STAT3/IL-6 signaling.

\section{Materials and methods}

Antibodies and cell culture reagents. Dulbecco's modified Eagle's medium (DMEM), penicillin-streptomycin solution, Trypsin-EDTA $(0.05 \%)$ and fetal bovine serum (FBS) were purchased from Gibco (Thermo Fisher Scientific, Inc.). Antibodies specific for $\beta$-actin (cat. no. sc-47778) and TLR-4 (cat.no. sc-293072) and secondary antibodies [goat anti-mouse (cat. no. sc-516102) and anti-rabbit (cat. no. sc-2357)] were obtained from Santa Cruz Biotechnology, Inc. The anti-IL-6 (cat. no. ab6672) and Tata-binding protein (TBP; cat. no. ab63766) antibodies were purchased from Abcam, and phosphorylated (p)-Jak2 (cat. no. 3776s), Jak2 (cat. no. 3230s), p-STAT3 (cat. no. 9145) and STAT3 (cat. no. 9139) antibodies were purchased from Cell Signaling Technology, Inc. NTS was donated by Nara Bio Co., Ltd. LPS (cat. no. L4391), and the STAT3 inhibitor 2-hydroxy-4-methylphenyl sulfonyl oxy acetyl amino-benzoic acid (cat. no. S3I-201) and TLR-4 inhibitor (TLR4-C34; cat. no. SML0832) were purchased from Sigma-Aldrich (Merck KGaA).

Cell culture and treatment. C2C12 myoblasts (cat. no. CRL-1772; American Type Culture Collection) were cultured in DMEM supplemented with $10 \%$ FBS and $1 \%$ penicillin-streptomycin at $37^{\circ} \mathrm{C}$ in $5 \% \mathrm{CO}_{2}$. For each experiment, cells at 70-80\% confluency cells were gently washed twice with phosphate-buffered saline (PBS). Unless otherwise specified, cells were treated with $0.2 \mu \mathrm{g} / \mathrm{ml}$ NTS in dimethyl sulfoxide (DMSO; cat. no. D8418; Sigma-Aldrich; Merck KGaA) for $24 \mathrm{~h}$ at $37^{\circ} \mathrm{C}$.

Cell viability assay. Cell viability was assessed using the 3-(4,5-dimethylthiazol-2-yl)-2,5-diphenyltetrazolium bromide (MTT; cat. no. M2128; Sigma-Aldrich; Merck KGaA) assay. Briefly, cells were re-suspended in DMEM 1 day prior to drug treatment at a density of $1 \times 10^{4}$ cells/well in 24 -well culture plates. The next day, the culture medium was replaced with fresh medium containing DMEM (vehicle control) or different concentrations of NTS $(0.1-2 \mu \mathrm{g})$, followed by incubation for $24 \mathrm{~h}$ at $37^{\circ} \mathrm{C}$. Following this treatment, MTT $(5 \mathrm{mg} / \mathrm{ml})$ was added and the culture plates were incubated at $37^{\circ} \mathrm{C}$ for $4 \mathrm{~h}$. The resulting formazan product was dissolved in DMSO and absorbances were measured at $560 \mathrm{~nm}$ using the Ultra Multifunctional Microplate Reader (Tecan US, Inc.). Cell viability was determined from these readings using the formula $\%$ viability $=$ (fluorescence value of MSM/fluorescence value of non-treated control) x100. All measurements were performed in triplicate and experiments were repeated at least three times. Cell viability was confirmed by WST-1 (Roche Diagnostics $\mathrm{GmbH}$ ) assay. Briefly, cells were re-suspended in DMEM 1 day prior to drug treatment at a density of $4 \times 10^{3}$ cells/well in 96-well culture plates. The next day, the culture medium was replaced with fresh medium containing DMEM (vehicle control) or different concentrations of NTS (0.1-2 $\mu \mathrm{g}$ ) and cells were incubated for $24 \mathrm{~h}$ at $37^{\circ} \mathrm{C}$. Following this treatment, $10 \mu \mathrm{l} /$ well cell proliferation Reagent WST-1 was added and the culture dishes were incubated at $37^{\circ} \mathrm{C}$ for $4 \mathrm{~h}$. Absorbances were measured at $460 \mathrm{~nm}$ using an Ultra Multifunctional Microplate Reader (Tecan US, Inc.). Cell viability was determined from these readings using the formula \% viability $=$ (fluorescence value of MSM/fluorescence value of non-treated control) x100. All measurements were performed in triplicate and experiments were repeated at least three times.

Apoptosis analysis. Fluorescein-conjugated Annexin V (Annexin V-FITC) was used to measure the apoptosis in C2C12 cells. The NTS or LPS treated cells were washed with PBS and re-suspended in a binding buffer at a concentration of $1 \times 10^{6}$ cells. Then, the cells were stained with Annexin V-FITC and PI for $10 \mathrm{~min}$ in the dark at room temperature. The percentage of apoptotic cells was measured by flow cytometry using a FACSCalibur flow cytometer (Bio-Rad Laboratories, Inc.) and the analysis was performed using FlowJo software v10 (FlowJo LLC). Apoptotic rate was calculated using live cells vs. dead cells, which included early apoptosis, late apoptosis or necrosis.

Western blotting. Whole cell lysates were prepared from untreated or NTS-treated C2C12 myoblasts by incubating the cells on ice with radioimmunoprecipitation lysis buffer (cat. no. 20-188; EMD Millipore) containing phosphatase and protease inhibitors. Cells were lysed by aspirating through a 23 -gauge needle and the lysate was centrifuged at $18,300 \mathrm{x}$ g for $10 \mathrm{~min}$ at $4^{\circ} \mathrm{C}$ to remove cellular debris. Protein concentrations were measured using the Bradford method (Thermo Fisher Scientific, Inc.). Equal amounts of protein (100 $\mu \mathrm{g}$ per lane) were subjected to $10 \%$ sodium dodecyl sulfate-polyacrylamide gel electrophoresis. Then, the separated proteins were transferred onto nitrocellulose membranes. The blots were blocked for $1 \mathrm{~h}$ with $5 \%$ skimmed milk (cat. no. 90002-594; BD Biosciences) in TBS with Tween-20 (TBST) buffer [20 mM Tris-HCl (cat. no. 10708976001; Sigma-Aldrich; Merck KGaA), pH 7.6, $137 \mathrm{mM} \mathrm{NaCl}$ (Formedium Ltd.) and 0.1X Tween-20 (cat. no. 0777; Scientific Sales, Inc.)]. Membranes were then probed overnight at $4^{\circ} \mathrm{C}$ with the indicated primary antibodies (1:1,000; anti-TLR-4, anti-IL-6, anti-p-Jak2, anti-Jak2, anti-p-STAT3, anti-STAT3 and anti- $\beta$-actin) diluted in $5 \%$ bovine serum albumin (BSA; EMD Millipore) or 5\% skimmed milk 
(Difco $^{\mathrm{TM}}$ skim milk; BD Biosciences). Membranes were then washed with TBST and incubated for 1-1.5 h at room temperature with horseradish peroxidase (HRP)-conjugated secondary antibodies (1:1,000). Detection was performed using the Enhanced Chemiluminescence Plus detection kit (Cytiva) and LAS-4000 imaging device (Version 1.0; Fujifilm Corporation). Blots were stripped with Restore Western Blot Stripping Buffer (Thermo Fisher Scientific, Inc.). Densitometry was performed using ImageJ software (version 1.8.0_172; National Institutes of Health).

Reverse transcription (RT)-semi-quantitative PCR. Total RNA was extracted from the cells using the RNeasy Mini kit (Qiagen $\mathrm{GmbH}$ ) according to the manufacturer's instructions. RNA was spectrophotometrically quantified at $260 \mathrm{~nm}$. Subsequently, RT-semi-quantitative PCR analyses were performed to detect TLR-4, IL-6 and GAPDH RNA expression. Briefly, cDNA was synthesized from total RNA by incubating the samples at $42^{\circ} \mathrm{C}$ for $1 \mathrm{~h}$ and then at $95^{\circ} \mathrm{C}$ for 5 min using a First-Strand cDNA Synthesis kit (cat. no. K-2041; Bioneer Corporation) and oligod(T) primers. The RT-PCR Premix kit (cat. no. K-2016; Bioneer Corporation) was used to amplify TLR-4, IL-6 and GAPDH with primers synthesized by Bioneer Corporation. To generate a 359-bp TLR-4 fragment, the following primers were used: TLR-4 sense, 5'-GCTTTCACCTCTGCCTTCAC-3' and antisense, 5'-CGAGGCTTTTCCATCCAATA-3'. To generate a 396-bp IL-6 fragment, the following primers were used: IL-6 sense, 5'-AGCCCTGAGAAAGGAGACAT-3' and antisense, 5'-CTGCGCAGAATGAGATGAGT-3'. Then, a 320-bp GAPDH mRNA fragment was generated with the following primers: GAPDH sense, 5'-AAGGCCATCACCATCTTCCA-3' and antisense, 5'-ACGATGCCAAAGTGGTCATG-3'. The PCR conditions for TLR-4 were as follows: $95^{\circ} \mathrm{C}$ for $5 \mathrm{~min}$, 32 cycles of $95^{\circ} \mathrm{C}$ for $60 \mathrm{sec}, 58^{\circ} \mathrm{C}$ for $60 \mathrm{sec}$ and $72^{\circ} \mathrm{C}$ for $60 \mathrm{sec}$ and $72^{\circ} \mathrm{C}$ for $10 \mathrm{~min}$. The PCR conditions for IL- 6 and GAPDH were as follows: $95^{\circ} \mathrm{C}$ for $5 \mathrm{~min}, 32$ cycles of $95^{\circ} \mathrm{C}$ for $60 \mathrm{sec}, 60^{\circ} \mathrm{C}$ for $60 \mathrm{sec}$ and $72^{\circ} \mathrm{C}$ for $60 \mathrm{sec}$ and $72^{\circ} \mathrm{C}$ for $10 \mathrm{~min}$. PCR products were resolved via electrophoresis on a $2 \%$ agarose gel and visualized with ethidium bromide (cat. no. E7637; Sigma-Aldrich; Merck KGaA) staining.

Flow cytometry analysis. After cultured cells were washed with cold PBS, cell pellets were isolated by centrifugation at $14,000 \mathrm{x}$ for $5 \mathrm{~min}$ at $4^{\circ} \mathrm{C}$ and incubated with $10 \%$ BSA on ice for $20 \mathrm{~min}$. Then, cells $\left(3 \times 10^{6}\right)$ were stained with the following antibodies (1:200) on ice for $30 \mathrm{~min}$ : PE CD282 (TLR4; cat. no. 312806; BioLegend, Inc.). Stained cells were washed with pre-cold PBS and flow cytometric analysis was performed using the FACSCalibur flow cytometer (BD Bioscience) and analysis was performed using FlowJo software v10 (FlowJo LLC).

Chromatin immunoprecipitation (ChIP) assay. The ChIP assay was performed using the Imprint ChIP kit (cat. no. CHP1; Sigma-Aldrich; Merck KGaA) according to the manufacturer's protocol. Briefly, C2C12 myoblasts were fixed by adding $1 \%$ formaldehyde to media containing cells immediately and quenched with $1.25 \mathrm{M}$ glycine at room temperature. Following washing with PBS, the cells were suspended in nuclei preparation buffer and shearing buffer (provided in ChIP kit) and sonicated under optimized conditions (20 sec on and off for 15 min with $25 \%$ amplitude on ice). The sheared DNA was then centrifuged at $16,000 \mathrm{x} \mathrm{g}$ for $10 \mathrm{~min}$ at $4^{\circ} \mathrm{C}$ and the cleared supernatant was used for protein/DNA immunoprecipitation. The clarified supernatant was diluted with buffer (1:1 ratio) and $5-\mu 1$ aliquots of the diluted samples were used as internal controls. Then, the diluted supernatant was incubated with anti-STAT3 antibody $(1: 1,000)$ in pre-coated wells for $90 \mathrm{~min}$ at $4^{\circ} \mathrm{C}$. The controls were incubated with normal goat $\operatorname{IgG}(1: 1,000)$ and then with anti-RNA polymerase II for $90 \mathrm{~min}$ at $4^{\circ} \mathrm{C}$. The unbound DNA was washed with immunoprecipitation wash buffer and the bound DNA was collected by the cross-link reversal method using a DNA release buffer containing proteinase $\mathrm{K}$. The released DNA and DNA from the internal control were purified using the GenElute Binding Column G (Sigma-Aldrich; Merck KGaA). The DNA was then quantified via RT-qPCR. The primer sequences were: IL-6 sense, 5'-GACTGAGCCTAAGGGTGCAT-3' and antisense, 5'-ACCACTAGAGGGCCAAGTCA-3'.

Nuclear protein extraction. Nuclear protein extracts were isolated using a nuclear extract kit (cat. no. AY2002; Panomics, Inc.). Briefly, cells were washed with PBS and buffer A containing DTT, Protease Inhibitor, Phosphates Inhibitor I and Phosphates Inhibitor II were added and incubated in ice on a rocking platform at $200 \mathrm{rpm}$ for $10 \mathrm{~min}$. The samples were transferred to micro centrifuge tubes and the supernatant removed by centrifugation at $14,000 \mathrm{x}$ g for $3 \mathrm{~min}$ at $4^{\circ} \mathrm{C}$. Then buffer $\mathrm{B}$ containing DTT, Protease Inhibitor, Phosphates Inhibitor I and Phosphates Inhibitor II were added and incubated on ice for $1 \mathrm{~h}$. The nuclear extract was collected by taking the supernatant by centrifugation at $14,000 \mathrm{x}$ g for $5 \mathrm{~min}$ at $4^{\circ} \mathrm{C}$ and western blotting was performed as described above for further analysis.

Enzyme-linked immunosorbent assay (ELISA). ELISA was performed for the quantitative detection of IL-6 using a mouse IL-6 ELISA kit (cat. no. ab100713; Abcam). C2C12 cells were treated with NTS for $24 \mathrm{~h}$ and with $100 \mathrm{ng} / \mathrm{ml}$ LPS for $4 \mathrm{~h}$ and the spent media were used for the assay. The samples were added to anti-mouse IL-6-coated microwells along with sample diluent and a biotin-conjugate solution. Following incubation, streptavidin-HRP was added and the plates were further incubated for $1 \mathrm{~h}$ in a shaker at room temperature. Then, 3,3',5,5'-tetramethylbenzidine solution was added after washing. Finally, a stop solution was added once the high-concentrated standard turned a dark blue color. The absorbance was read with a microplate reader at $450 \mathrm{~nm}$ and calculations were performed according to the assay protocol.

Statistical analyses. All experiments were performed at least three times. Results are expressed as the mean \pm standard error of the mean. Statistical analyses were conducted using the one-way analysis of variance (ANOVA) or unpaired Student's t-test. One-way ANOVA was followed by Tukey's post hoc test. Analyses were performed using the SAS 9.3 program (SAS Institute, Inc.). $\mathrm{P}<0.05$ was considered to indicate a statistically significant difference.

\section{Results}

Protective effect of NTS on LPS-induced loss of cell viability. To examine whether NTS exhibits a protective effect on cell 
A

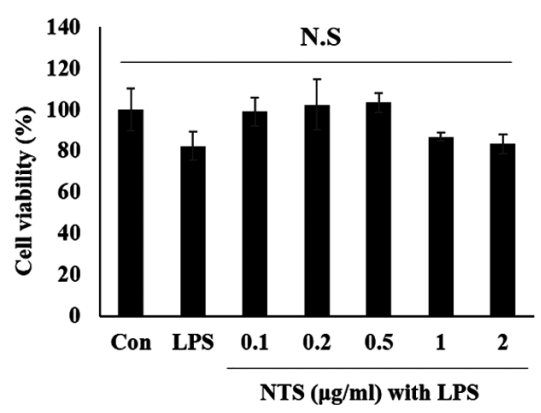

B

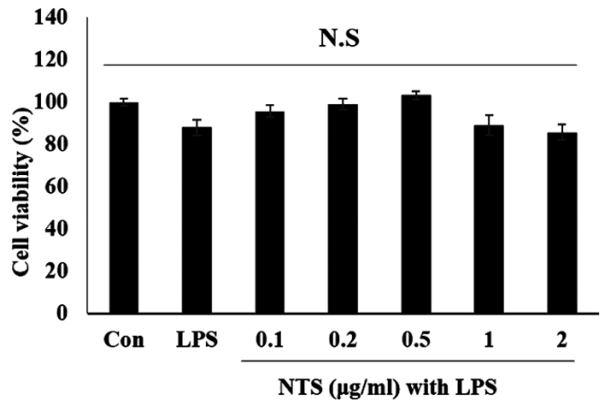

D
C

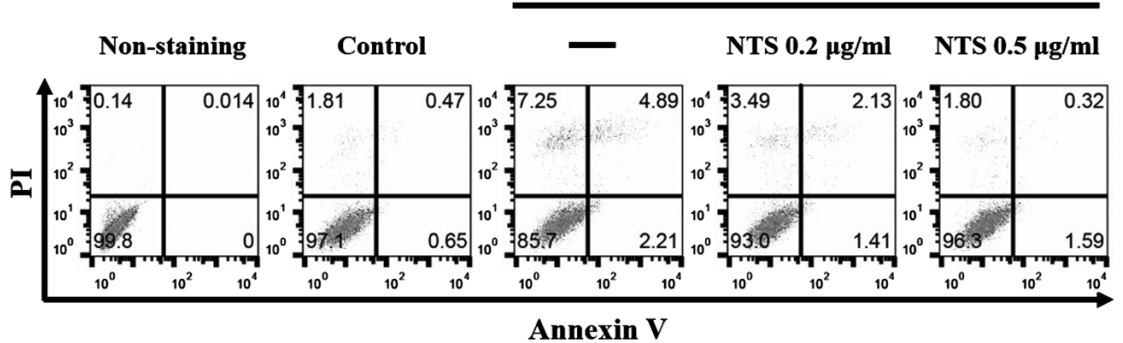

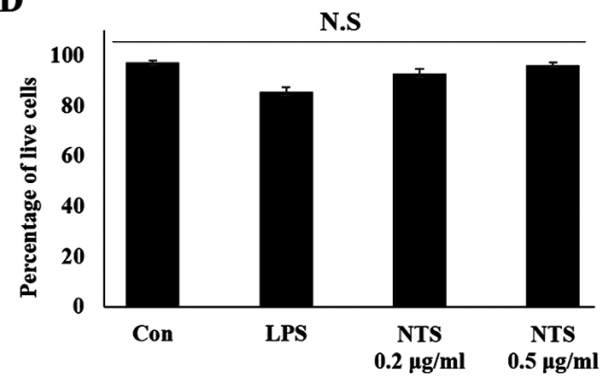

Figure 1. Effect of NTS on the viability of LPS-treated C2C12 cells. (A) MTT assay was performed to evaluate viability of C2C12 cells following treatment with or without $100 \mathrm{ng} / \mathrm{ml}$ LPS or $0.1-2 \mu \mathrm{g} / \mathrm{ml}$ NTS for $24 \mathrm{~h}$. Cell viability was determined using the formula $\%$ viability $=($ fluorescence value of methylsulfonylmethane/fluorescence value of non-treated control) x100. Data are representative of three independent experiments. Non-significant results were observed via statistical analysis. (B) WST-1 assay showing the cyto protective effects of NTS against LPS-induced cell death. Data are representative of three independent experiments. Non-significant results were observed via statistical analysis. (C) Flow cytometry analysis using Annexin V and PI staining in C2C12 cells following treatment with LPS and NTS for $24 \mathrm{~h}$. (D) Graphical representation of the percentage of cells undergoing phases of apoptosis following treatment with $100 \mathrm{ng} / \mathrm{ml}$ LPS or 0.2 and $0.5 \mu \mathrm{g} / \mathrm{ml}$ NTS in C2C12 myoblasts. The values are presented as mean \pm standard error of the mean of three independent experiments performed in triplicate $(n=3)$. NTS, non-toxic sulfur; LPS, lipopolysaccharide; Con, control; N.S., non-significant.

viability, the MTT assay (Fig. 1A) and WST-1 assay (Fig. 1B) were performed. It was found that LPS induced $\sim 20 \%$ of cell death during a treatment period of $24 \mathrm{~h}$. To determine the optimum concentration of NTS, cells were treated with $0.1,0.2,0.5,1$ and $2 \mu \mathrm{g} / \mathrm{ml} \mathrm{NTS}$ in the presence of $100 \mathrm{ng} / \mathrm{ml}$ LPS. A non-significant increase in cell numbers with $0.1,0.2$ and $0.5 \mu \mathrm{g} / \mathrm{ml}$ NTS was observed, whereas 1 and $2 \mu \mathrm{g} / \mathrm{ml}$ NTS decreased cell viability. In order to confirm the cyto protective effect of NTS, flow cytometry analysis was conducted in $\mathrm{C} 2 \mathrm{C} 12$ myoblasts (Fig. 1C). The results confirmed an increase in cell proliferation compared with LPS-treated cells (Fig. 1D). The pro-apoptotic factor BAX protein was also analyzed and this confirmed the cyto protective effect of NTS against LPS-induced cell death (Fig. S1). From these results, 0.2 and $0.5 \mu \mathrm{g} / \mathrm{ml}$ NTS were selected for further studies.

NTS inhibits LPS-induced mRNA expression of TLR4 and IL-6. From the cell viability assay, the optimal concentrations of NTS were determined to be 0.2 and $0.5 \mu \mathrm{g} / \mathrm{ml}$. Subsequently, it was determined whether NTS can inhibit LPS-induced expression of the inflammatory receptor TLR-4 and pro-inflammatory cytokine IL-6. C2C12 cells were treated with LPS or NTS and then mRNA was isolated from these cells to analyze the expression of TLR-4 and IL-6 via RT-semi-quantitative PCR (Fig. 2A). The obtained results suggested an increase in the expression of TLR-4 and IL-6 with LPS treatment, which was inhibited by NTS treatment (Fig. 2B). These results suggested the anti-inflammatory effect of NTS.

NTS downregulates LPS-induced JAK2/STAT3/IL-6 signaling. It was observed that NTS inhibited LPS-induced mRNA expression of TLR-4 and IL-6 mRNA. Therefore, the protein expression of TLR-4 and JAK2/STAT3/IL-6 was measured with western blotting. The obtained results demonstrated an increase in the expression of TLR-4 and JAK2/STAT3/IL-6 in LPS-treated cells, which was downregulated by $0.5 \mu \mathrm{g} / \mathrm{ml}$ NTS (Fig. 3A) without affecting the expression of total JAK2 and STAT3. To determine the role of TLR-4 and STAT3 in the anti-inflammatory effect of NTS, cells were treated with a TLR-4 inhibitor (TLR4-C34) or STAT3 inhibitor S3I-201 $(100 \mu \mathrm{M})$. It was observed that the TLR-4 inhibitor demonstrated a similar significant pattern to NTS; by contrast, the STAT3 inhibitor inhibited the expression of p-STAT3 and IL-6. These results provided evidence of the role of TLR-4 and STAT3 in the anti-inflammatory activity of NTS (Fig. 3B). The ratio of phosphorylated proteins to their total form also demonstrated similar result to the phosphorylated proteins compared with $\beta$-actin (Fig. 3C). In addition, the role of TLR-4 in inflammation was confirmed by examining LPS-induced expression of TLR-4 and its inhibition by NTS treatment via flow cytometry analysis (Fig. 3D).

NTS inhibits expression of nuclear $p$-STAT3 and its binding to the IL-6 promoter. The present study demonstrated that NTS inhibited LPS-induced expression of TLR-4, JAK2, STAT3 and IL-6. It was hypothesized that STAT3 would translocate from the cytoplasm to the nucleus to bind to the promoters of pro-inflammatory cytokines in LPS-induced inflammation. Therefore, C2C12 cells were treated with or without LPS or NTS for $24 \mathrm{~h}$ and nuclear proteins were isolated to analyze the expression of STAT3 via western blotting. The expression of p-STAT3 increased in LPS-treated cells, whereas total 
A

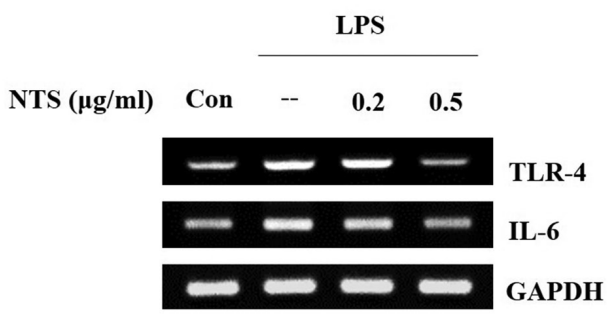

B
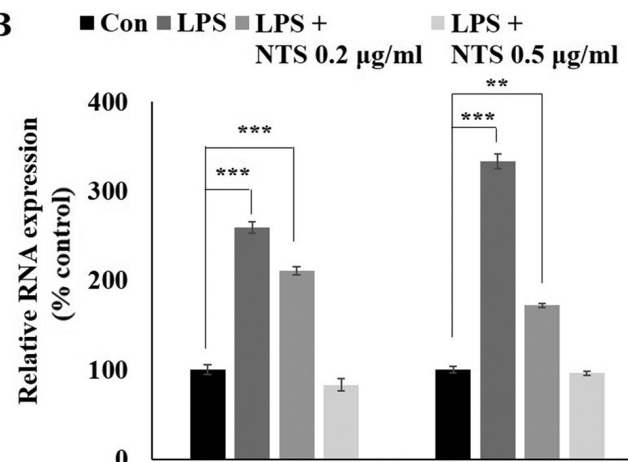

NTS $0.2 \mu \mathrm{g} / \mathrm{ml} \quad \mathrm{NTS} 0.5 \mu \mathrm{g} / \mathrm{ml}$

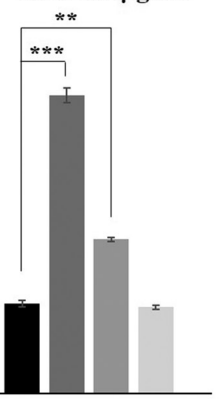

IL-6

Figure 2. NTS inhibits TLR-4 and IL-6 mRNA expression. (A) Reverse transcription-semi-quantitative PCR results showing the analysis of TLR-4 and IL-6 mRNA expression following the treatment of C2C12 cells with $100 \mathrm{ng} / \mathrm{ml}$ LPS or 0.2 and $0.5 \mu \mathrm{g} / \mathrm{ml}$ NTS for $24 \mathrm{~h}$. (B) Relative mRNA expression levels of TLR-4 and IL-6 were determined via densitometry and were normalized to GAPDH mRNA. Data are representative of three independent experiments. ${ }^{* *} \mathrm{P}<0.01$ and ${ }^{* * *} \mathrm{P}<0.001$ vs. control. NTS, non-toxic sulfur; TLR-4, Toll-like receptor 4; LPS, lipopolysaccharide; Con, control.

A

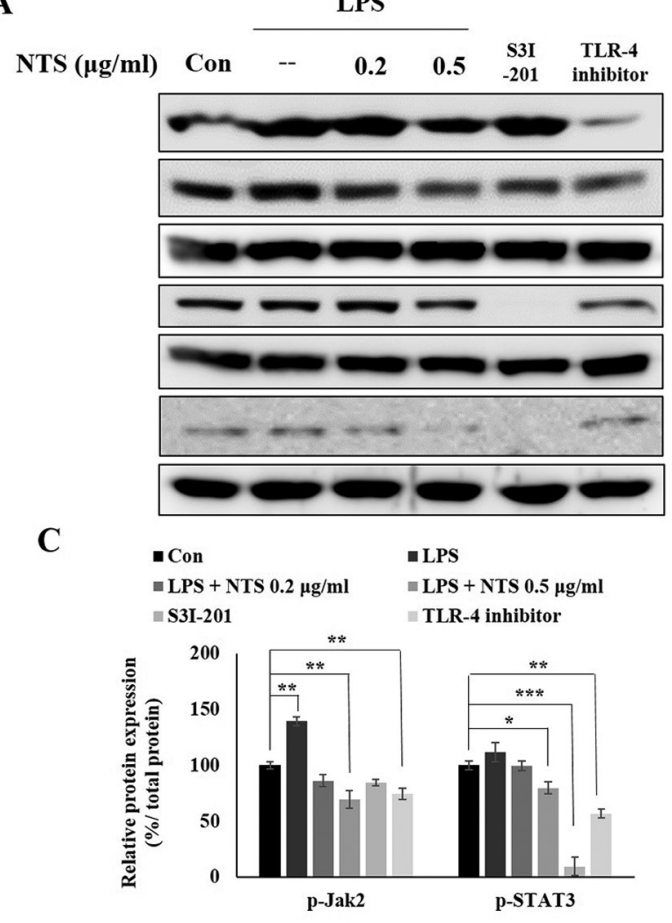

B

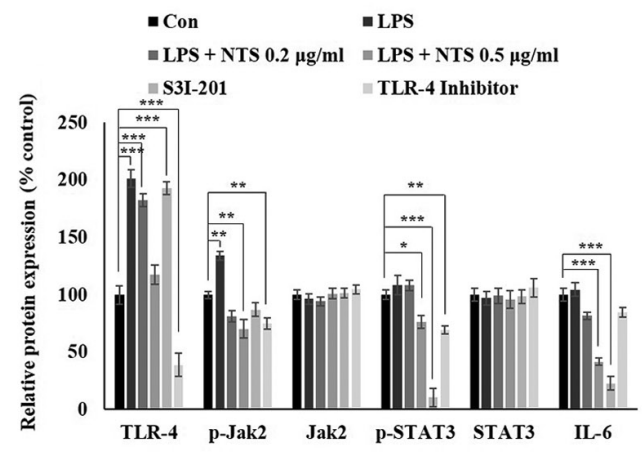

D
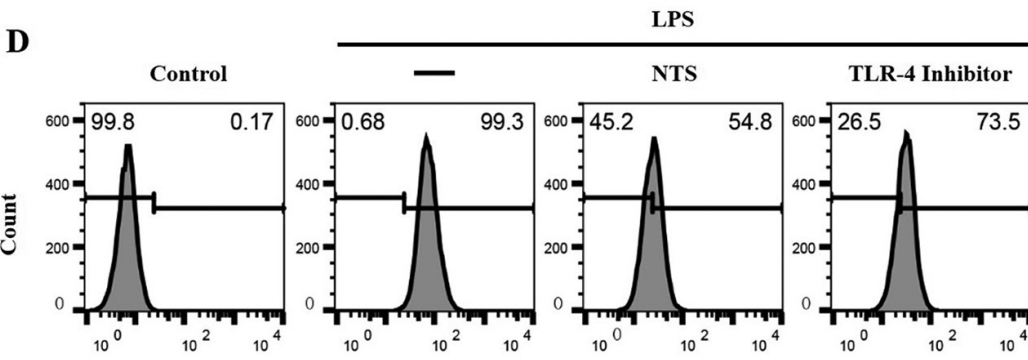

TLR-4 (95 kDa)

p-Jak2 (125 kDa)

Jak2 (130 kDa)

p-STAT3 (86 kDa)

STAT3 (86 kDa)

IL-6 (25 kDa)

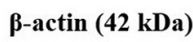
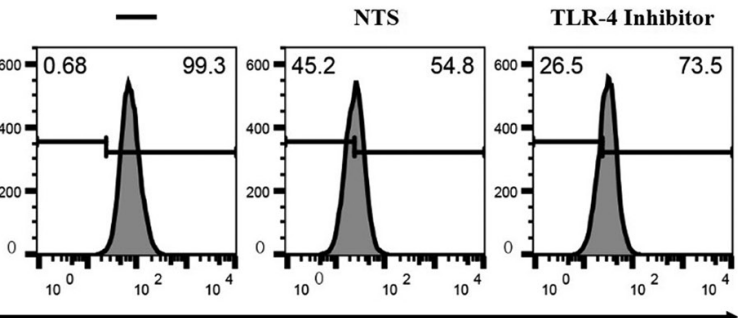

Figure 3. NTS inhibits TLR-4, JAK2, STAT3 and IL-6 signaling. (A) Western blotting showing the expression of TLR-4, JAK2, p-JAK2, STAT3, p-STAT3 and IL- 6 proteins following the treatment of C2C12 cells with $100 \mathrm{ng} / \mathrm{ml}$ LPS and 0.2 or $0.5 \mu \mathrm{g} / \mathrm{ml}$ NTS or $100 \mu \mathrm{M}$ S3I-201 or $80 \mu \mathrm{M}$ TLR4-C34 for $24 \mathrm{~h}$ (B) Relative protein expression levels of TLR-4, JAK2, p-JAK2, STAT3, p-STAT3 and IL-6 were determined via densitometry and were normalized to $\beta$-actin. Data are representative of three independent experiments. (C) Relative expression levels of p-JAK2 and p-STAT3 were determined via densitometry and were normalized to total JAK2 and STAT3. (D) Flow cytometry (fluorescence-activated cell sorting) analysis of TLR-4 expression in C2C12 cells showing the expression levels of LPS-induced TLR-4 and its inhibition by $0.5 \mu \mathrm{g} / \mathrm{ml} \mathrm{NTS}$ or $80 \mu \mathrm{M}$ Toll-like receptor 4 inhibitor (TLR4-C 34 ) for $24 \mathrm{~h}$. ${ }^{*} \mathrm{P}<0.05,{ }^{* *} \mathrm{P}<0.01$ and ${ }^{* * *} \mathrm{P}<0.001$ vs. control. NTS, non-toxic sulfur; TLR-4, Toll-like receptor 4; JAK2, Janus kinase 2; p-, phosphorylated; LPS, lipopolysaccharide; Con, control.

STAT3 levels remained unchanged in non-treated control cells (Fig. 4A). NTS inhibited the expression of p-STAT3 without affecting the expression levels of STAT3, indicating the possible inhibition of the nuclear translocation of STAT3 by NTS (Fig. 4B). The ratio of nuclear p-STAT3 protein to total nuclear STAT3 protein also demonstrated a similar result to the p-STAT3 expression compared with TBP (Fig. 4C). It was hypothesized that nuclear p-STAT3 may bind to the IL-6 promoter. To confirm this, the expression of the STAT3-IL-6 complex was analyzed by ChIP assay (Fig. 4D). The result demonstrated a significant increase in LPS-induced expression of the STAT3-IL-6 complex, which was downregulated by $0.5 \mu \mathrm{g} / \mathrm{ml} \mathrm{NTS} \mathrm{(Fig.} \mathrm{4E).}$

NTS inhibits LPS-induced STAT3-dependent IL-6 expression. The inhibition of the STAT3-IL-6 complex by NTS was demonstrated in LPS-induced inflammation. The present study assessed whether NTS could inhibit IL-6 release into the media. C2C12 cells were treated with or without LPS or NTS and the media was collected to conduct a mouse IL-6 ELISA. 
A

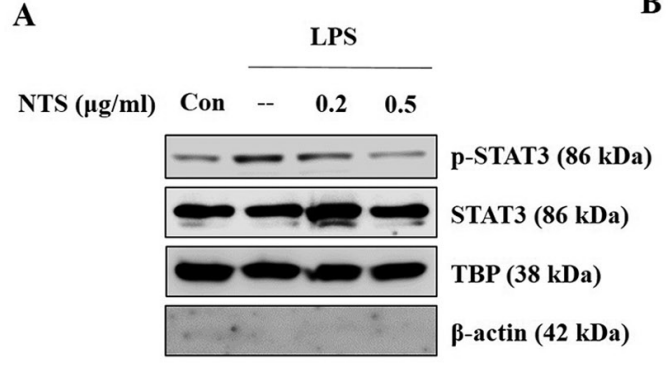

D

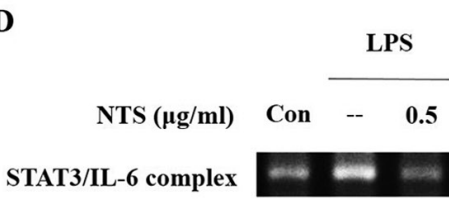

B

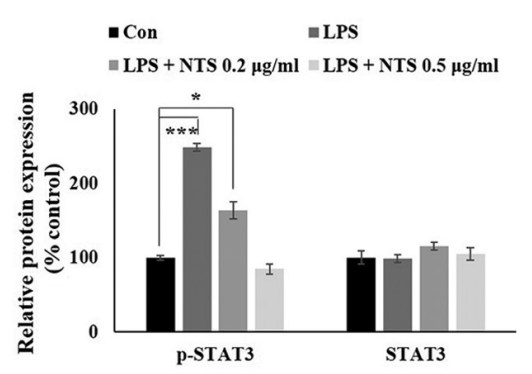

C

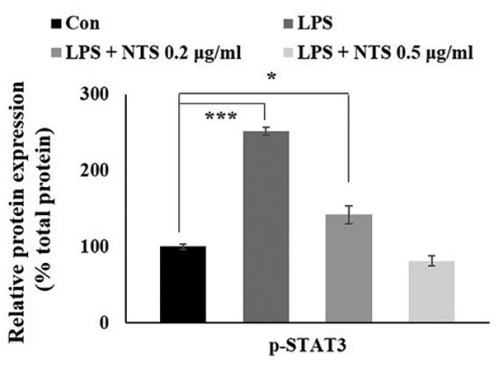

$\mathbf{E}$

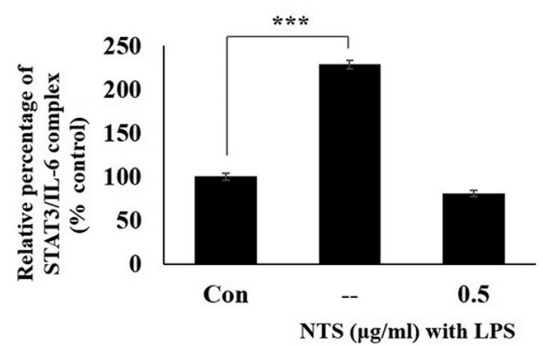

Figure 4. NTS inhibits the expression levels of nuclear p-STAT3 and its binding to the IL-6 promoter. (A) Western blotting of the nuclear extracts of C2C12 cells showing the expression of STAT3 and p-STAT3 nuclear proteins following treatment with $100 \mathrm{ng} / \mathrm{ml} \mathrm{LPS} \mathrm{and} 0.2 \mathrm{or} 0.5 \mu \mathrm{g} / \mathrm{ml} \mathrm{NTS}$ for $24 \mathrm{~h}$. (B) Relative expression levels of STAT3 and p-STAT3 nuclear proteins were determined via densitometry and normalized to TBP. Data are representative of three independent experiments. (C) Relative expression levels of nuclear p-STAT3 determined via densitometry and were normalized to total STAT3. (D) ChIP analysis of STAT3-IL-6 complex formation in C2C12 cells following treatment with $100 \mathrm{ng} / \mathrm{ml} \mathrm{LPS}$ or $0.5 \mu \mathrm{g} / \mathrm{ml} \mathrm{NTS}$ for $24 \mathrm{~h}$. Data were quantified using reverse transcription-semi-quantitative PCR. (E) Relative STAT3-IL-6 complex binding obtained in C2C12 cells with $100 \mathrm{ng} / \mathrm{ml} \mathrm{LPS}$ or $0.5 \mu \mathrm{g} / \mathrm{ml} \mathrm{NTS}$ for $24 \mathrm{~h}$ by ChIP assay and expressed as a percentage of the control. Data are representative of three independent experiments. ${ }^{*} \mathrm{P}<0.05$ and ${ }^{* * *} \mathrm{P}<0.001$ vs. control. NTS, non-toxic sulfur; p-, phosphorylated; LPS, lipopolysaccharide; ChIP, chromatin immunoprecipitation; Con, control; TBP, Tata-binding protein.

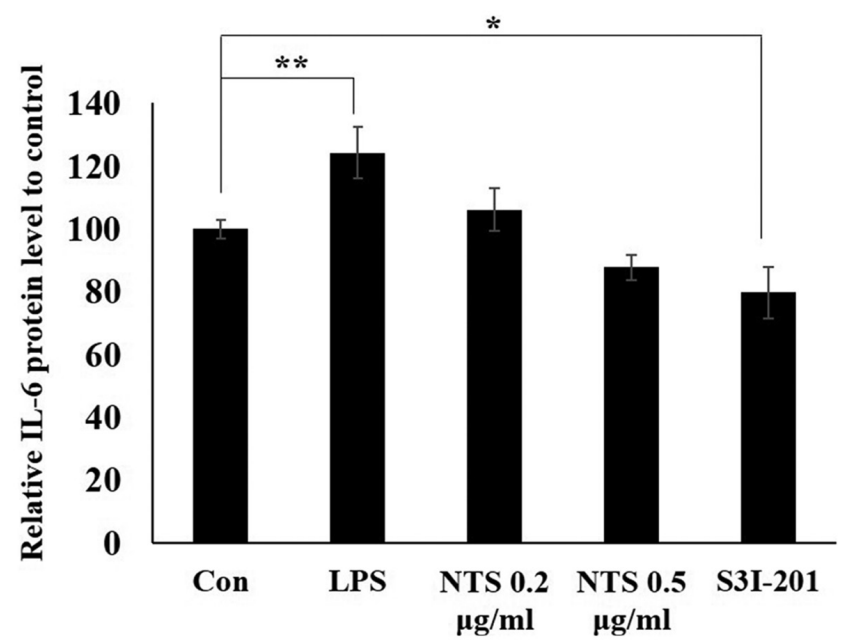

Figure 5. NTS inhibits STAT3-dependent IL6 expression. ELISA analysis showing the inhibition of LPS-induced expression of IL-6 following treatment with 0.2 and $0.5 \mu \mathrm{g} / \mathrm{ml}$ of NTS or $100 \mu \mathrm{M} \mathrm{S3I}-201$ for $24 \mathrm{~h}$. Data are representative of three independent experiments. $\mathrm{P}<0.05$ and ${ }^{* *} \mathrm{P}<0.01$ vs. control. NTS, non-toxic sulfur; Con, control; LPS, lipopolysaccharide.

The result demonstrated a significant increase in LPS-induced IL-6 expression, whereas NTS decreased IL-6 expression (Fig. 5). A similar pattern was observed in the STAT3 inhibitor-treated group. These results demonstrated that NTS exhibited an anti-inflammatory effect by regulating STAT3. Taken together, NTS inhibited LPS-induced inflammation by inhibiting LPS-induced expression of TLR-4, JAK2, STAT3 and IL-6. It also inhibited the expression of IL-6 mRNA to induce its anti-inflammatory effects (Fig. 6).

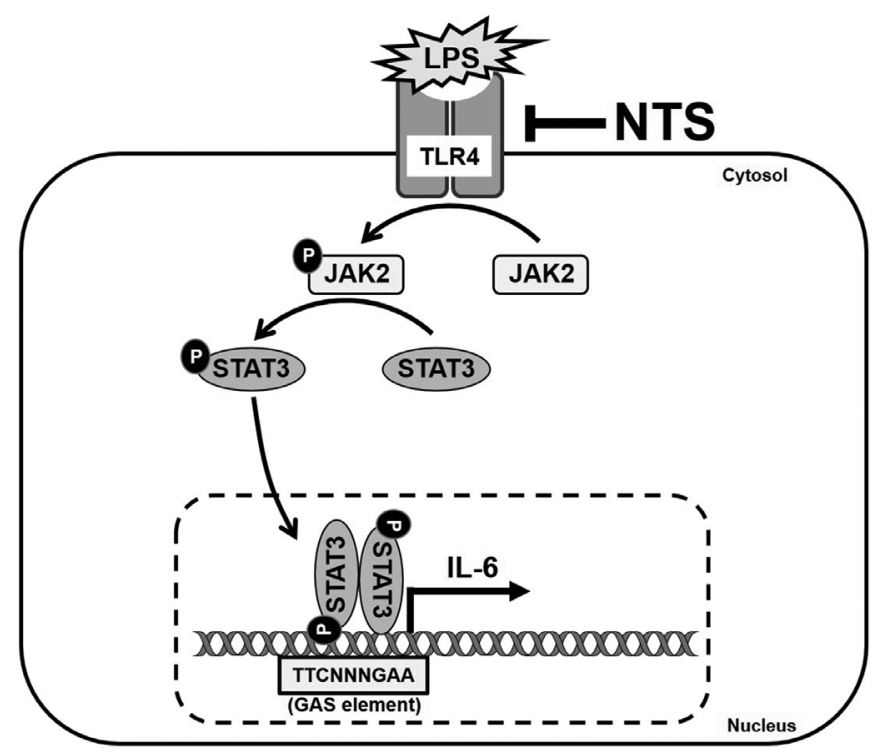

Figure 6. Molecular regulatory mechanism of LPS-induced inflammatory responses by TLR- 4 and JAK2/STAT3 via IL- 6 signaling. The anti-inflammatory activities of NTS are mediated by inhibiting these pathways and the binding of STAT3 to the promoter of the pro-inflammatory cytokine IL-6. LPS, lipopolysaccharide; NTS, non-toxic sulfur; TLR-4, Toll-like receptor 4; JAK2, Janus kinase 2; p-, phosphorylated; GAS, $\gamma$-interferon activation site.

\section{Discussion}

The treatment of inflammation with naturally active compounds is a promising strategy as a number of natural compounds exhibit anti-inflammation activities with fewer side effects (26). After calcium and phosphorus, sulfur is suggested to be the 
most abundant mineral element present in the human body that is used for normal maintenance and it is generally derived from the sources of dietary protein because of the toxicity associated with direct administration (27). Sulfur-containing amino acids are essential for optimal growth and protein synthesis (28). Sulfur-containing compounds possess various activities, including anticancer (29), anti-inflammatory (30) and antioxidant activity (31). NTS is considered a good source as it enables the supplementation of the non-toxic form of sulfur. If NTS were able to inhibit LPS-induced inflammation, then it could be considered a candidate drug for the treatment of inflammation.

If a natural compound protects against LPS-induced cell death by increasing the cell viability of a normal cell, then it can be considered that the particular concentration of that natural compound is not associated with significant adverse effects. The present study used $\mathrm{C} 2 \mathrm{C} 12$ mouse myoblasts for anti-inflammatory studies as these cells are used for inflammation studies. Inflammation by LPS can activate the ubiquitin-proteasome pathway via TLR4 and induces catabolism in cultured $\mathrm{C} 2 \mathrm{C} 12$ (32). These $\mathrm{C} 2 \mathrm{C} 12$ myoblasts are commonly used for the regulation of LPS-induced inflammation by candidate drugs in vitro $(33,34)$. However, the lack of a more relevant cell model could be considered as a limitation of this study. The results demonstrated that LPS induced $20 \%$ of cell death in $\mathrm{C} 2 \mathrm{C} 12$ cells, whereas concentrations $>0.5 \mu \mathrm{g} / \mathrm{ml}$ NTS demonstrated a protective effect on $\mathrm{C} 2 \mathrm{C} 12$ cells by increasing cell viability compared with LPS treatment. Inhibition in apoptosis by NTS also gave evidence for our previous study that demonstrated NTS can act as a growth hormone by inducing growth hormone signaling (25). Therefore, from the results of the cell viability and apoptosis assay, it was hypothesized that $0.5 \mu \mathrm{g} / \mathrm{ml}$ NTS could inhibit LPS-induced inflammation.

To examine the anti-inflammatory activity of NTS, the effect of NTS on LPS-induced expression of the inflammation response receptor TLR-4 and pro-inflammatory cytokine IL-6 was initially assessed. TLR-4 is considered one of the main receptors triggering inflammatory responses (35). The addition of LPS leads to the activation of TLR- 4 by binding to cluster of differentiation (CD)14 that is anchored to raft proteins (36). IL-6 is a key factor in inflammation response pathways and it takes part in both non-classical inflammation pathways and Hippo pathways (37). It also serves an important role in maintaining the delicate balance between inflammation and immune resolution (38). The results of the present study demonstrated an increase in the expression of TLR-4 and IL-6 with LPS treatment, whereas NTS decreased their expression at both transcriptional and translational levels and their levels in spent media. These results suggested the role of NTS as an effective anti-inflammatory drug against LPS-induced inflammation.

The JAK/STAT pathway is one of the key signaling pathways as a target for autoimmune diseases and inflammation $(39,40)$. JAK/STAT signals promote inflammation by regulating the development of innate lymphoid cells in immune responses (41). It is the major pathway in inflammation that acts via signaling from TLR-4 in natural compound-based anti-inflammatory activity (42). The present study demonstrated that LPS inhibition induced the expression of TLR-4. In addition, it found that NTS inhibited LPS-induced expression of activated JAK2 and STAT3 without affecting the total forms of these proteins. Therefore, the results suggested that the anti-inflammatory activity of NTS was mediated via TLR-4 and JAK2/STAT3 signaling. STAT3 is a transcription factor for the pro-inflammatory cytokine IL-6 during inflammation (43). The results of the present study demonstrated that NTS inhibited the nuclear levels of activated STAT3 and its binding to the promoter region of IL- 6 , indicating that the inflammatory response via STAT3/IL-6 signaling was downregulated by NTS treatment. In particular, it blocks the transcription of IL-6, thereby inhibiting inflammation.

In conclusion, the present study demonstrated that the natural sulfur-containing compound NTS inhibited LPS-induced inflammation by modulating TLR-4 and JAK2/STAT3 signaling pathways. NTS also reduced the nuclear p-STAT3 protein levels and inhibits its binding to the promoter region of the pro-inflammatory cytokine IL-6. Taken together, the results suggested that NTS could be a potential candidate drug for the treatment of inflammation.

\section{Acknowledgements}

Not applicable.

\section{Funding}

The present study was supported by Nara Bio Co.,Ltd., Republic of Korea, in 2018 and by the Cooperative Research Program for Agriculture Science and Technology Development (project no. PJ01325702) and by the National Research Foundation of Korea (NRF) grant funded by the Korean government (MSIT; grant no. 2018R1C1B6006146).

\section{Availability of data and materials}

The datasets used and/or analyzed during the present study are available from the corresponding author on reasonable request.

\section{Authors' contributions}

YMY and KJJ designed the experiments. DYK, NS and ESJ performed most of the experiments. AR, HDK, IK, JCP and SWB helped with experiments and discussions. YMY, KJJ, DYK and NS analyzed the data. NS and DYK wrote the manuscript. HDK and IHK from Bio Co., Ltd., provided NTS and took part in project design and evaluation. DYK and NS confirm the authenticity of all the raw data. All authors helped to revise the manuscript and approved the final version for publication.

\section{Ethics approval and consent to participate}

Not applicable.

\section{Patient consent for publication}

Not applicable.

\section{Competing interests}

Hyoung Do Kim is affiliated with Nara Bio Co., Ltd., which provided funding for this study and supplied non-toxic sulfur. The remaining authors declare that they have no competing interests. 


\section{References}

1. Chen L, Deng H, Cui H, Fang J, Zuo Z, Deng J, Li Y, Wang X and Zhao L: Inflammatory responses and inflammation-associated diseases in organs. Oncotarget 9: 7204-7218, 2017.

2. Catorce MN and Gevorkian G: LPS-induced murine neuroinflammation model: Main features and suitability for pre-clinical assessment of nutraceuticals. Curr Neuropharmacol 14: 155-164, 2016.

3. Heinbockel L, Weindl G, Martinez-de-Tejada G, Correa W, Sanchez-Gomez S, Bárcena-Varela S, Goldmann T, Garidel P, Gutsmann T and Brandenburg K: Inhibition of lipopolysaccharideand lipoprotein-induced inflammation by antitoxin peptide Pep19-2.5 Front Immunol 9: 1704, 2018.

4. Kawai T and Akira S: Signaling to NF-kappaB by Toll-like receptors. Trends Mol Med 13: 460-469, 2007.

5. Liu T, Zhang L, Joo D and Sun SC: NF-кB signaling in inflammation. Signal Transduct Target Ther 2: 2, 2017.

6. Yamakawa T, Eguchi S, Matsumoto T, Yamakawa Y, Numaguchi K, Miyata I, Reynolds CM, Motley ED and Inagami T: Intracellular signaling in rat cultured vascular smooth muscle cells: Roles of nuclear factor-kappaB and p38 mitogen-activated protein kinase on tumor necrosis factor-alpha production. Endocrinology 140: 3562-3572, 1999.

7. Olson CM,Hedrick MN, Izadi H, Bates TC, Olivera ER and Anguita J: p38 mitogen-activated protein kinase controls NF-kappaB transcriptional activation and tumor necrosis factor alpha production through RelA phosphorylation mediated by mitogen- and stress-activated protein kinase 1 in response to Borrelia burgdorferi antigens. Infect Immun 75: 270-277, 2007.

8. Ngkelo A, Meja K, Yeadon M, Adcock I and Kirkham PA: LPS induced inflammatory responses in human peripheral blood mononuclear cells is mediated through NOX4 and Gi $\alpha$ dependent PI-3kinase signalling. J Inflamm (Lond) 9: 1, 2012.

9. Nipin SP, Darvin P, Yoo YB, Joung YH, Kang DY, Kim DN, Hwang TS, Kim SY, Kim WS, Lee HK, et al: The combination of methylsulfonylmethane and tamoxifen inhibits the Jak2/STAT5b pathway and synergistically inhibits tumor growth and metastasis in ER-positive breast cancer xenografts. BMC Cancer 15: 474, 2015.

10. Okugawa S, Ota Y, Kitazawa T, Nakayama K, Yanagimoto S, Tsukada K, Kawada M and Kimura S: Janus kinase 2 is involved in lipopolysaccharide-induced activation of macrophages. Am J Physiol Cell Physiol 285: C399-C408, 2003.

11. Lee JJ, Kim DH, Kim DG, Lee HJ, Min W, Rhee MH, Cho JY, Watarai $\mathrm{M}$ and Kim S: Toll-like receptor 4-linked Janus kinase 2 signaling contributes to internalization of Brucella abortus by macrophages. Infect Immun 81: 2448-2458, 2013.

12. Kasembeli MM, Bharadwaj U, Robinson P and Tweardy DJ: Contribution of STAT3 to inflammatory and fibrotic diseases and prospects for its targeting for treatment. Int J Mol Sci 19: 19, 2018.

13. Darnell JE Jr, Kerr IM and Stark GR: Jak-STAT pathways and transcriptional activation in response to IFNs and other extracellular signaling proteins. Science 264: 1415-1421, 1994.

14. Moran A, Akcan Arikan A, Mastrangelo MA, Wu Y, Yu B, Poli V and Tweardy DJ: Prevention of trauma and hemorrhagic shock-mediated liver apoptosis by activation of stat3alpha. Int J Clin Exp Med 1: 213-247, 2008.

15. Moran A, Tsimelzon AI, Mastrangelo MA, Wu Y, Yu B, Hilsenbeck SG, Poli V and Tweardy DJ: Prevention of trauma/hemorrhagic shock-induced lung apoptosis by IL-6-mediated activation of Stat3. Clin Transl Sci 2: 41-49, 2009.

16. Chen WD, Zhang JL, Wang XY, Hu ZW and Qian YB: The JAK2/ STAT3 signaling pathway is required for inflammation and cell death induced by cerulein in AR42J cells. Eur Rev Med Pharmacol Sci 23: 1770-1777, 2019.

17. Wang SW and Sun YM: The IL-6/JAK/STAT3 pathway: Potential therapeutic strategies in treating colorectal cancer (Review). Int J Oncol 44: 1032-1040, 2014.

18. Chung YC and Chang YF: Serum interleukin-6 levels reflect the disease status of colorectal cancer. J Surg Oncol 83: 222-226, 2003.

19. van der Merwe M and Bloomer RJ: The influence of methylsulfonylmethane on inflammation-associated cytokine release before and following strenuous exercise. J Sports Med (Hindawi Publ Corp) 2016: 7498359, 2016.

20. Kim YH, Kim DH, Lim H, Baek DY, Shin HK and Kim JK: The anti-inflammatory effects of methylsulfonylmethane on lipopolysaccharide-induced inflammatory responses in murine macrophages. Biol Pharm Bull 32: 651-656, 2009.

21. Koh E and Surh J: Influence of sulfur fertilization on the antioxidant activities of onion juices prepared by thermal treatment. Prev Nutr Food Sci 21: 160-164, 2016
22. Caron JM, Bannon M, Rosshirt L, Luis J, Monteagudo L, Caron JM and Sternstein GM: Methyl sulfone induces loss of metastatic properties and reemergence of normal phenotypes in a metastatic cloudman S-91 (M3) murine melanoma cell line.PLoS One 5: el1788, 2010.

23. Lim CI, Choe HS, Kang C, Lee BK and Ryu KS: Effects of dietary organic sulfur on performance, egg quality and cell-mediated immune response of laying hens. Korean J Poult Sci 45: 97-107, 2018.

24. Lee JS, Kwon JK, Han SH and An IJ: Toxicity study of detoxication sulphur at 3 months post-treatment in rats. J Fd Hyg Saf 25: 263-268, 2010.

25. Kang DY, Sp N, Jo ES, Kim HD, Kim IH, Bae SW, Jang KJ and Yang YM: Non toxic sulfur enhances growth hormone signaling through the JAK2/STAT5b/IGF 1 pathway in C2C12 cells. Int J Mol Med 45: 931-938, 2020.

26. Kang DY,Darvin P, Yoo YB, Joung YH, Sp N, Byun HJ and Yang YM: Methylsulfonylmethane inhibits HER2 expression through STAT5b in breast cancer cells. Int J Oncol 48: 836-842, 2016.

27. Nimni ME, Han B and Cordoba F: Are we getting enough sulfur in our diet? Nutr Metab (Lond) 4: 24, 2007.

28. Griffith OW: Mammalian sulfur amino acid metabolism: An overview. Methods Enzymol 143: 366-376, 1987.

29. Lim EJ, Hong DY, Park JH, Joung YH, Darvin P, Kim SY, Na YM, Hwang TS, Ye SK, Moon ES, et al: Methylsulfonylmethane suppresses breast cancer growth by down-regulating STAT3 and STAT5b pathways. PLoS One 7: e33361, 2012.

30. Sousa-Lima I, Park SY, Chung M, Jung HJ, Kang MC, Gaspar JM, Seo JA, Macedo MP, Park KS, Mantzoros C, et al: Methylsulfonylmethane (MSM), an organosulfur compound, is effective against obesity-induced metabolic disorders in mice. Metabolism 65: 1508-1521, 2016.

31. Henrotin Y and Mobasheri A: Natural products for promoting joint health and managing osteoarthritis. Curr Rheumatol Rep 20: 72, 2018

32. Doyle A, Zhang G, Abdel Fattah EA, Eissa NT and Li YP: Toll-like receptor 4 mediates lipopolysaccharide-induced muscle catabolism via coordinate activation of ubiquitin-proteasome and autophagy-lysosome pathways. FASEB J 25: 99-110, 2011.

33. Liu S, Adewole D, Yu L, Sid V, Wang B, O K and Yang C: Rutin attenuates inflammatory responses induced by lipopolysaccharide in an in vitro mouse muscle cell (C2C12) model. Poult Sci 98: 2756-2764, 2019.

34. Baker LA, Martin NRW, Kimber MC, Pritchard GJ, Lindley MR and Lewis MP: Resolvin E1 (Rv E1 ) attenuates LPS induced inflammation and subsequent atrophy in $\mathrm{C} 2 \mathrm{C} 12$ myotubes. J Cell Biochem 119: 6094-6103, 2018.

35. Rogero MM and Calder PC: Obesity, inflammation, Toll-like receptor 4 and fatty acids. Nutrients 10: 10, 2018.

36. Płóciennikowska A, Hromada-Judycka A, Borzęcka $\mathrm{K}$ and Kwiatkowska K: Co-operation of TLR4 and raft proteins in LPS-induced pro-inflammatory signaling. Cell Mol Life Sci 72: 557-581, 2015.

37. Yeung YT, Aziz F, Guerrero-Castilla A and Arguelles S: Signaling pathways in inflammation and anti-inflammatory therapies. Curr Pharm Des 24: 1449-1484, 2018.

38. Taams LS: Inflammation and immune resolution. Clin Exp Immunol 193: 1-2, 2018.

39. Banerjee S, Biehl A, Gadina M, Hasni S and Schwartz DM: Erratum to: JAK-STAT signaling as a target for inflammatory and autoimmune diseases: Current and future prospects. Drugs 77: 1261, 2017.

40. Banerjee S, Biehl A, Gadina M, Hasni S and Schwartz DM: JAK-STAT signaling as a target for inflammatory and autoimmune diseases: Current and future prospects. Drugs 77: 521-546, 2017.

41. Stabile H, Scarno G, Fionda C, Gismondi A, Santoni A, Gadina M and Sciumè G: JAK/STAT signaling in regulation of innate lymphoid cells: The gods before the guardians. Immunol Rev 286: 148-159, 2018.

42. Zhou GY, Yi YX, Jin LX, Lin W, Fang PP, Lin XZ, Zheng Y and Pan CW: The protective effect of juglanin on fructose-induced hepatitis by inhibiting inflammation and apoptosis through TLR4 and JAK2/STAT3 signaling pathways in fructose-fed rats. Biomed Pharmacother 81: 318-328, 2016.

43. Zimmers TA, Fishel ML and Bonetto A: STAT3 in the systemic inflammation of cancer cachexia. Semin Cell Dev Biol 54: 28-41, 2016.

This work is licensed under a Creative Commons Attribution-NonCommercial-NoDerivatives 4.0 International (CC BY-NC-ND 4.0) License. 